reasons for visit and site of infection. Cases positive from the rectum alone were compared with cases positive from urethra, cervix, vault and urine alone or in multiple sites including the rectum. CT testing was conducted with Genprobe Aptima by the Provincial Laboratory for Public Health. Univariate analysis was completed using Chi-square or Fisher's exact test and Mann-Whitney for continuous variables. Bivariate logistic regression, adjusted for gender, was completed using significant $(P>0.05)$ at the univariate level.

Results Twenty percent of all CT cases $(n=245)$ were diagnosed in the rectum only; females were more likely to be diagnosed with rectal-only CT $(24.6 \%)$ than males $(16.6 \% ; P=0.001)$. No cases of rectal-only CT were found among heterosexual men; therefore regression models were completed for women and men who have sex with men (MSM). Factors associated with rectal-only CT for women included older age (AOR $=1.05,95 \% \mathrm{CI}: 1.02$, 1.08), being tested at Clinic A (AOR = 3.0, 95\% CI: 1.8, 5.1), and being named as a contact to an STI (AOR $=0.3,95 \%$ CI: 0.1, 0.9). For MSM, being asymptomatic ( $\mathrm{AOR}=2.2,95 \% \mathrm{CI}$ : $1.2,4.1)$ remained significant.

Conclusions After the switch to NAAT testing for rectal CT, additional cases of CT were found among women and MSM. Differences between clinics are likely attributable to different screening practises for women.

\section{P3.017 CHLAMYDIA TRACHOMATIS REPEAT TESTING IN AUSTRALIA}

doi:10.1136/sextrans-2013-051184.0477

\begin{abstract}
${ }^{12} \mathbf{M}$ Lim, ${ }^{3} \mathrm{~W}$ Dimech, ${ }^{1,2} \mathrm{C}$ Van Gemert, ${ }^{4} \mathrm{D}$ Boyle, 'D McCarthy, ${ }^{1} \mathrm{E}$ Weaver, ${ }^{5} \mathrm{~B}$ Donovan, 1,2M Hellard. 'Burnet Institute, Melbourne, Australia; ${ }^{2}$ Monash University, Melbourne, Australia; ${ }^{3} N R L$, Melbourne, Australia; ${ }^{4}$ University of Melbourne, Melbourne, Australia; ${ }^{5}$ Kirby Institute, University of New South UK, Sydney, Australia
\end{abstract}

Background Current guidelines recommend that sexually active people aged under 25 are screened annually for Chlamydia. Those testing positive should be retested around 12 weeks later to detect re-infection. The Australian Collaboration for Chlamydia Enhanced Sentinel Surveillance (ACCESS) Laboratory Network has collected chlamydia testing data from 15 Australian public and private laboratories since 2008 . This study reviews the frequency of repeat testing for $\mathrm{C}$. trachomatis.

Methods Chlamydia test results and associated demographic data were extracted from participating laboratories' information systems, de-identified with a non-reversible unique code and sent to a central database using GRHANITE ${ }^{\circledR}$ software. Using the unique identifier, cases of multiple testing episodes from individuals were reviewed to determine the frequency of repeat testing.

Results 641,302 chlamydia test results were collected from 547,761 individuals during the calendar years 2008-2010; 49,655 (7.7\%) were positive. Overall, $9.6 \%$ individuals had multiple testing episodes, increasing to $23.4 \%$ among those with an initially positive result. The mean number of testing episodes per individual was 1.11 (range 1-29) and mean time between repeat tests was 201 days following negative samples but 95 days after a positive sample. Among individuals who had a repeat test, for those with a negative result $19.6 \%$ of repeat tests were performed within 42 days, $42.8 \%$ within 120 days and $86.0 \%$ within 13 months. This is compared with $41.9 \%$ ( $\leq 42$ days), $76.6 \%$ ( $\leq 120$ days) and $96.6 \%$ ( $\leq 13$ months) for repeat tests following an initially positive result.

Conclusion Individuals with positive test results were found to be re-tested more frequently and earlier than those with negative test results. However, less than one quarter of individuals who tested positive for chlamydia were re-tested and over $40 \%$ of these were re-tested too soon after initial diagnosis ( $<6$ weeks), risking a false positive test result.

\section{P3.018 DEVELOPMENT OF A C. TRACHOMATIS-SPECIFIC COMPETITIVE PGP3 ELISA}

doi:10.1136/sextrans-2013-051184.0478

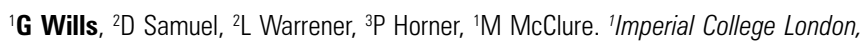
London, UK; ${ }^{2}$ Health Protection Agency, London, UK; ${ }^{3}$ University of Bristol, Bristol, UK

Background Chlamydia trachomatis (CT) DNA testing of genital samples principally from symptomatic persons provides information about active infection only, and is unlikely to represent true prevalence of current and past infection in the population. Serological tests applied to serum collections that are more representative of the general population can help understanding the pattern of the infection. We previously described an indirect immunoglobulin G (IgG) enzyme-linked immunosorbent assay (ELISA) based on the CT-specific antigen Pgp3. Sensitivity and specificity were determined using ROC curve analysis of data from 356 sera from CTinfected patients and 722 paediatric sera. The assay works particularly well in women, with a greater sensitivity $(74 \%)$ than commercial assays $(60 \%)$, and is suitable for use in seroprevalence studies. However, there is a need to confirm the specificity of samples reactive in the indirect Pgp3 ELISA and, to this end, we have developed a competitive Pgp3 ELISA.

Methods Purified IgG from human sera containing high titre antibody to CT was labelled with HRP and, by optimising conditions and using chequerboard titrations, an assay developed where test sera compete with labelled IgG for epitopes on the Pgp3 protein.

Results The competitive assay was optimised, then 89 sera from our CT-infected patient cohort (patients having had at least one positive CT NAAT result at least one month previously) and 91 paediatric sera were assayed by both the indirect and competitive Pgp3 ELISAs. Results by these two assays were concordant.

Conclusion A competitive ELISA based on the CT-specific Pgp3 protein has been developed, which confirms the specificity of the indirect Pgp3 ELISA.

\section{P3.019* IS CONCURRENCY, NUMBER OF PARTNERS OR DURATION OF PARTNERSHIP THE MOST IMPORTANT FACTOR ASSOCIATED WITH CHLAMYDIA IN YOUNG AUSTRALIAN ADULTS?}

doi:10.1136/sextrans-2013-051184.0479

${ }^{1}$ A Yeung, ${ }^{2} \mathrm{M}$ Temple-Smith, ${ }^{1} \mathrm{~A}$ Bingham, ${ }^{3,4} \mathrm{C}$ Fairley, ${ }^{5} \mathrm{M}$ Law, ${ }^{5} \mathrm{R}$ Guy, ${ }^{6} \mathrm{~N}$ Low, ${ }^{5} \mathrm{~B}$ Donovan, $5 \mathrm{~J}$ Kaldor, '1 Hocking. 'Centre for Women's Health, Gender \& Society, Melbourne School of Population and Global Health, Univ, Melbourne, Australia, ${ }^{2}$ Department of General Practice, University of Melbourn, Melbourne, Australia, ${ }^{3}$ Melbourne Sexual Health Centre, Melbourne, Australia, "Melbourne School of Population and Global Health, University of Melbourne, Melbourne, Australia, ${ }^{5}$ Kirby

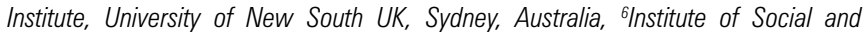
Preventive Medicine, University of Bern, Bern, Switzerland

Background There is considerable discussion about which sexual behaviour variables are most strongly associated with chlamydia. We investigated this in a study conducted within a chlamydia screening trial.

Methods A consecutive sample of patients aged 16-29 attending 134 GP clinics in 54 postcodes was recruited. Patients completed a questionnaire and chlamydia test. Using random effects logit regression models we estimated (1) the significance of a variable's association with chlamydia (likelihood ratio test for model fit), and; (2) the strength of association with chlamydia (odds ratio[OR]). Number of partners in the last 12 months and partnership duration (years) were fitted as continuous variables. Each model included age, gender and a sexual behaviour variable. A multivariate model including all sexual behaviour variables was also run. All analyses accounted for intra-cluster correlation within postcode. 